\title{
Cardiovascular and arousal responses to single-lumen endotracheal and double-lumen endobronchial intubation in the normotensive and hypertensive elderly
}

\author{
Kyung Yeon Yoo ${ }^{1}$, Cheol Won Jeong ${ }^{1}$, Woong Mo Kim', Hyung Kon Lee ${ }^{1}$, Seongtae Jeong ${ }^{1}$, Seok Jae \\ $\mathrm{Kim}^{1}$, Hong Beum Bae ${ }^{1}$, Dong Yun Lim, and Sung Su Chung ${ }^{2}$ \\ ${ }^{1}$ Department of Anesthesiology and Pain Medicine, Chonnam National University Medical School, ${ }^{2}$ Chonnam National University \\ School of Dentistry, Gwangju, Korea
}

Background: Endotracheal intubation usually causes transient hypertension and tachycardia. The cardiovascular and arousal responses to endotracheal and endobronchial intubation were determined during rapid-sequence induction of anesthesia in normotensive and hypertensive elderly patients.

Methods: Patients requiring endotracheal intubation with (HT, $n=30)$ or without hypertension $(\mathrm{NT}, \mathrm{n}=30)$ and those requiring endobronchial intubation with $(\mathrm{HB}, \mathrm{n}=30)$ or without hypertension $(\mathrm{NB}, \mathrm{n}=30)$ were included in the study. Anesthesia was induced with intravenous thiopental $5 \mathrm{mg} / \mathrm{kg}$ followed by succinylcholine $1.5 \mathrm{mg} / \mathrm{kg}$. After intubation, all subjects received $2 \%$ sevoflurane in $50 \%$ nitrous oxide and oxygen. Mean arterial pressure (MAP), heart rate (HR), plasma catecholamine concentration, and Bispectral Index (BIS) values, were measured before and after intubation.

Results: The intubation significantly increased MAP, HR, BIS values and plasma catecholamine concentrations in all groups, the peak value of increases was comparable between endotracheal and endobronchial intubation. However, pressor response persisted longer in the HB group than in the HT group $(5.1 \pm 1.6$ vs. $3.2 \pm 0.9 \mathrm{~min}, \mathrm{P}<0.05)$. The magnitude of increases in MAP and norepinephrine from pre-intubation values was greater in the hypertensive than in the normotensive group $(\mathrm{P}<0.05)$, while there were no differences in those of HR and BIS between the hypertensive and normotensive groups.

Conclusions: Cardiovascular response and arousal response, as measured by BIS, were similar in endobronchial and endotracheal intubation groups regardless of the presence or absence of hypertension except for prolonged pressor response in the HB group. However, the hypertensive patients showed enhanced cardiovascular responses than the normotensive patients. (Korean J Anesthesiol 2011; 60: 90-97)

Key Words: Bispectral index, Cardiovascular effects, Catecholamine, Endobronchial intubation, Endotracheal intubation, Hypertension.

Received: April 30, 2010. Revised: 1st, May 30, 2010; 2nd, June 1, 2010. Accepted: August 8, 2010.

Corresponding author: Kyung Yeon Yoo, M.D., Department of Anesthesiology and Pain Medicine, Chonnam National University Medical School, 8, Hak-1 dong, Dong-gu, Gwangju 501-757, Korea. Tel: 82-62-220-6893, Fax: 82-62-232-6294, E-mail: kyyoo@jnu.ac.kr

(c) This is an open-access article distributed under the terms of the Creative Commons Attribution Non-Commercial License (http:// creativecommons.org/licenses/by-nc/3.0/), which permits unrestricted non-commercial use, distribution, and reproduction in any medium, provided the original work is properly cited. 


\section{Introduction}

Laryngoscopy and endotracheal intubation often results in increases of arterial pressure, heart rate (HR) and circulating catecholamines $[1,2]$ as well as an arousal response on the electroencephalograph (EEG) during induction of anesthesia [3]. The intubation may be accomplished by single-lumen tubes (SLT) or double-lumen tubes (DLT). The main portion of DLT is placed in the lower trachea approximately $1-2 \mathrm{~cm}$ above the carina, with the bronchial extension inserted far enough into one of the main bronchi. The cardiovascular response to airway stimulation differs in its magnitude at different sites within the airway [4]. Therefore, the cardiovascular response to DLT may differ from that to SLT. Many patients who require endobronchial intubation with the use of DLT are elderly, who have a diminished physiological reserve, alteration in autonomic function and an increased incidence of coexisting cardiovascular diseases such as coronary atherosclerosis and hypertension $[5,6]$. The cardiovascular responses to tracheal intubation may be exaggerated in the hypertensive patient [1]. Moreover, the elderly exhibit an increased sensitivity to opioids and anesthetic drugs $[7,8]$. Therefore, they are more prone to develop myocardial ischemia, stroke, cardiac arrhythmias, or sudden death during such abrupt hemodynamic changes that occur in endotracheal or endobronchial intubation [9].

Thompson et al. [10] have found that the pressor response to endobronchial intubation is of a similar magnitude to that known to occur in response to endotracheal intubation in previous studies [2]. However, to date, no study has directly compared the cardiovascular response to endotracheal and endobronchial intubation in patients undergoing general anesthesia. Moreover, although laryngoscopy and endotracheal intubation is associated with an arousal response during induction of anesthesia [3], the degree of arousal responses to endobronchial intubation has not been documented.

The present study aimed to compare the cardiovascular and arousal responses to SLT and DLT intubation in elderly patients with or without hypertension. The bispectral index (BIS) was used to identify the arousal response.

\section{Materials and Methods}

We prospectively studied 60 American Society of Anesthesiologists (ASA) physical status I and II requiring endotracheal intubation using SLT and 60 patients requiring endobronchial intubation using a left DLT with or without hypertension. An approval of the Institutional Ethics Committee and written informed consents from the subjects were obtained. Patients were eligible to be in the normotensive group if they had no history of hypertension and the blood pressure on three occasions was $<160 \mathrm{mmHg}$ systolic and $<95 \mathrm{mmHg}$ diastolic. Patients were eligible to be in the hypertensive group if they had a history of hypertension with ongoing treatment and the admission blood pressure on three occasions was $<180 \mathrm{mmHg}$ systolic and $<105 \mathrm{mmHg}$ diastolic. Criteria for exclusion were: age $<60$ yr old; ASA physical status III or greater; blood pressure $>180 \mathrm{mmHg}$ systolic and $>105 \mathrm{mmHg}$ diastolic; obesity (body mass index $>30 \mathrm{~kg} / \mathrm{m}^{2}$ ); anticipated difficulty with airway maintenance or intubation; recent myocardial infarction, congestive cardiac failure or electrocardiographic (EKG) evidence of heart block; or the presence of a cardiac pacemaker.

Patients were classified into one of the following four groups according to the technique of intubation, and the presence or absence of hypertension: those requiring endotracheal intubation without hypertension (NT group, $n=30$ ), those requiring endobronchial intubation without hypertension (NB group, $\mathrm{n}=30$ ), those requiring endotracheal intubation with hypertension (HT group, $\mathrm{n}=30$ ), and those requiring endobronchial intubation with hypertension (HB group, $\mathrm{n}=$ 30).

All patients were premedicated with $0.1 \mathrm{mg} / \mathrm{kg}$ midazolam orally $60 \mathrm{~min}$ before induction of anesthesia. The hypertensive received their usual antihypertensive drugs on the day of surgery. Before arrival in the operating room, an intravenous (i.v.) catheter was placed to allow administration of i.v. fluids and medications. In addition, a 20-G catheter was inserted into a radial artery to measure arterial pressure and for blood sampling. Heart rate was determined from ECG traces. A standard BIS monitor strip (BIS Sensor ${ }^{\circledR}$; Aspect Medical Systems, USA) was applied to the forehead before induction of anesthesia, and BIS was measured throughout the study using a BIS monitor (MODEL a-2000; 3.1 software version, Aspect Medical Systems, USA). The anesthesiologist who participated in the anesthetic care was blinded to the BIS value. For each patient, a rest period of at least $30 \mathrm{~min}$ was allowed to elapse before starting the protocol.

After breathing 100\% oxygen, anesthesia was induced at 1.5 min before initiating laryngoscopy and intubation with i.v. thiopental $5 \mathrm{mg} / \mathrm{kg}$ given over $20 \mathrm{~s}$ followed by succinylcholine $1.5 \mathrm{mg} / \mathrm{kg}$ i.v. given over $5 \mathrm{~s}$, while the patients were ventilated with $100 \%$ oxygen. One and half minutes after induction (time 0 ), when neuromuscular block was achieved, laryngoscopy and intubation were performed using a SLT (Sheridan ${ }^{\circledR}$, Teleflex Medical Inc, Mexico) or a left DLT (Broncho-Cath ${ }^{\mathrm{TM}}$, Mallinckrodt Medical Inc, Ireland) by a skilled anesthesiologist. After intubation, all patients were mechanically ventilated with sevoflurane ( $2 \%$ inspired) in $50 \%$ nitrous oxide and oxygen throughout the study to maintain end-tidal carbon dioxide between 35 and $40 \mathrm{mmHg}$ by adjusting the tidal volume (8$10 \mathrm{ml} / \mathrm{kg}$ ) and respiratory frequency ( $8-12$ breaths/min). The 
fresh gas flow rate was adjusted to $6 \mathrm{~L} / \mathrm{min}$ throughout the study. Muscle relaxation was maintained with rocuronium given as an initial bolus of $0.8 \mathrm{mg} / \mathrm{kg}$ within a few minutes of succinylcholine administration. The size of the left DLT was chosen based on the measurement of the left bronchial diameter on chest radiograph, as described by Hannallah et al. [11]. Proper placement of the DLT was confirmed by using fiberoptic bronchoscopy when the study had concluded because bronchoscopy itself may stimulate the airway. A humidifier (Thermovent $600^{\circledR}$, Portex Inc, UK) was used. The duration of laryngoscopy (defined as the time from the beginning of laryngoscopy to inflation of the cuff) and any difficulties in laryngoscopy or intubation were noted. Data from patients in whom intubation required longer than $60 \mathrm{~s}$ were excluded.

Arterial pressure (mmHg), HR (beats/min) and BIS values were record immediately before induction of anesthesia (baseline, time $-1.5 \mathrm{~min}$ ), just before initiating laryngoscopy and intubation (pre-intubation, time 0), and at 1-min intervals for 5 min after the intubation. The maximum BIS values displayed every minute were taken by downloading data from the electronic memory of the monitor at the end of surgery. Arousal response (defined by an increase in BIS to intubation) was determined by the differences of BIS values between peak values observed after intubation (for $3 \mathrm{~min}$ after intubation) and those just before starting laryngoscopy. Ephedrine was administered in $4 \mathrm{mg}$ increments for hypotension (systolic arterial blood pressure $[\mathrm{SAP}]<90 \mathrm{mmHg}$, or a decrease of $>30 \%$ from baseline values for more than $60 \mathrm{~s}$ ), and atropine in $0.5 \mathrm{mg}$ increments for bradycardia (HR less than 50 beats/min for more than $60 \mathrm{~s}$ ). For hypertension (SAP $>200 \mathrm{mmHg}$, or an increase of $>30 \%$ above baseline for $>60 \mathrm{~s}$ ) or tachycardia (HR $>120$ beats/min for $>60 \mathrm{~s}$ ) associated with intubation, the inspired sevoflurane concentration was increased by increments of $1 \%$. The time period it took for MAP and HR to recover to their baseline values following the intubation were recorded to define the periods during which the pressor and tachycardiac responses were observed, respectively.

Arterial blood samples were drawn before induction of anesthesia (baseline), just prior to laryngoscopy and intubation (pre-intubation), and 1,2, and 5 min after the onset of intubation. The samples were collected into prechilled tubes containing ethylenediamine tetraacetic acid-sodium and immediately centrifuged at 3,000 revolutions per minute for $10 \mathrm{~min}$ at $4^{\circ} \mathrm{C}$. The plasma was stored at $-70^{\circ} \mathrm{C}$ until assayed for catecholamine concentrations. Plasma concentrations of norepinephrine and epinephrine were measured in duplicates using high-pressure liquid chromatography [12]. The assay sensitivity was $10.0 \mathrm{pg} / \mathrm{ml}$, and within-run precision coefficients of variation were $13.5 \%$ for norepinephrine and $14.2 \%$ for epinephrine. All patients were interviewed for recall on the on the first postoperative day using a standardized set of postoperative questions [13], which included inquiring for the recall of any unpleasant dreams during intubation or surgery as well as explicit memory recall for operative events.

The sample size calculation was based on the primary endpoint of mean arterial pressure (MAP) value at tracheal intubation. In our pilot study, the maximum MAP in the NB group was $140 \pm 13 \mathrm{mmHg}$. A power analysis suggested a sample size of 27 patients in each group should be adequate to detect a $10 \mathrm{mmHg}$ difference in peak MAP at intubation with a two-sided significance level $\alpha$ of 0.05 and a power of 0.8 . Taking into account possible dropouts, we aimed to recruit about 30 patients in each group.

Data were expressed as number or mean \pm SD. They were analyzed using the statistical package (StatView ${ }^{\circledR}$ software version 4.0, Abacus Concepts Inc, USA) on a Macintosh computer. Sex was analyzed using Fisher's exact test. Other patient characteristic data were compared using a one-way analysis of variance. Serial changes in cardiovascular, hormonal, and BIS data were analyzed using two-way analysis of variance with repeated measures, with time as within-group factor group (endobronchial/endotracheal) as between-group factor, and the interaction between time and group also compared. A difference among the groups in BIS increase at intubation from the value measured just before starting laryngoscopy to peak value (arousal) was analyzed using analysis of variance. The Scheffé test was used for multiple pairwise comparisons when a significant difference was indicated with analysis of variance. Complication rates among the groups were analyzed by using the $\chi^{2}$ test where appropriate. A P value of $<0.05$ was considered statistically significant.

\section{Results}

There were no significant differences among the groups with respect to sex ratio, age, weight, and height (Table 1). The time required to accomplish endobronchial intubation was significantly longer than that for endotracheal intubation ( $\sim 23$ s vs. $\sim 14$ s, $\mathrm{P}<0.001)$ with or without hypertension. Antihypertensive medications did not differ between the two hypertensive groups (Table 1).

MAP and HR are illustrated in Fig. 1. Basal MAP and HR did not differ between endotracheal and endobronchial intubation groups in the normotensive (NT and NB) and hypertensive groups (HT and HB). HR was comparable among the groups. The induction of anesthesia with thiopental decreased MAP similarly in all groups. The intubation then significantly increased MAP from pre-intubation values in all groups, the magnitude of which did not differ between endotracheal and 
Table 1. Patient Characteristics

\begin{tabular}{|c|c|c|c|c|}
\hline & \multicolumn{2}{|c|}{ Normotensive } & \multicolumn{2}{|c|}{ Hypertensive } \\
\hline & $\mathrm{NT}(\mathrm{n}=30)$ & $\mathrm{NB}(\mathrm{n}=30)$ & $\mathrm{HT}(\mathrm{n}=30)$ & $\mathrm{HB}(\mathrm{n}=30)$ \\
\hline $\operatorname{Sex}(M / F)$ & $17 / 13$ & $21 / 9$ & $15 / 15$ & $22 / 8$ \\
\hline Age (yr) & $68 \pm 5$ & $67 \pm 5$ & $69 \pm 4$ & $67 \pm 7$ \\
\hline Weight (kg) & $60 \pm 9$ & $59 \pm 8$ & $60 \pm 10$ & $67 \pm 11$ \\
\hline Height (cm) & $159 \pm 9$ & $163 \pm 8$ & $158 \pm 9$ & $164 \pm 7$ \\
\hline Hemoglobin (g/dl) & $12.8 \pm 1.8$ & $12.6 \pm 1.6$ & $12.8 \pm 1.8$ & $13.3 \pm 1.4$ \\
\hline Intubation time (s) & $14 \pm 4$ & $22 \pm 6^{*, \dagger}$ & $14 \pm 4$ & $23 \pm 7^{*, \dagger}$ \\
\hline \multicolumn{5}{|l|}{ Preoperative medication } \\
\hline Calcium channel blockers & - & - & 20 & 22 \\
\hline$\beta$-blockers & - & - & 4 & 4 \\
\hline ACE inhibitors & - & - & 4 & 7 \\
\hline ARBs & - & - & 9 & 5 \\
\hline
\end{tabular}

Values are mean \pm SD or number of patients. NT: endotracheally intubated normotensives, NB: endobronchially intubated normotensives, HT: endotracheally intubated hypertensives, HB: endobronchially intubated hypertensives, ACE: angiotensin converting enzyme, ARBs: angiotensin II receptor blockers. ${ }^{*} \mathrm{P}<0.05$ vs. NT, ${ }^{\dagger} \mathrm{P}<0.05$ vs. HT.
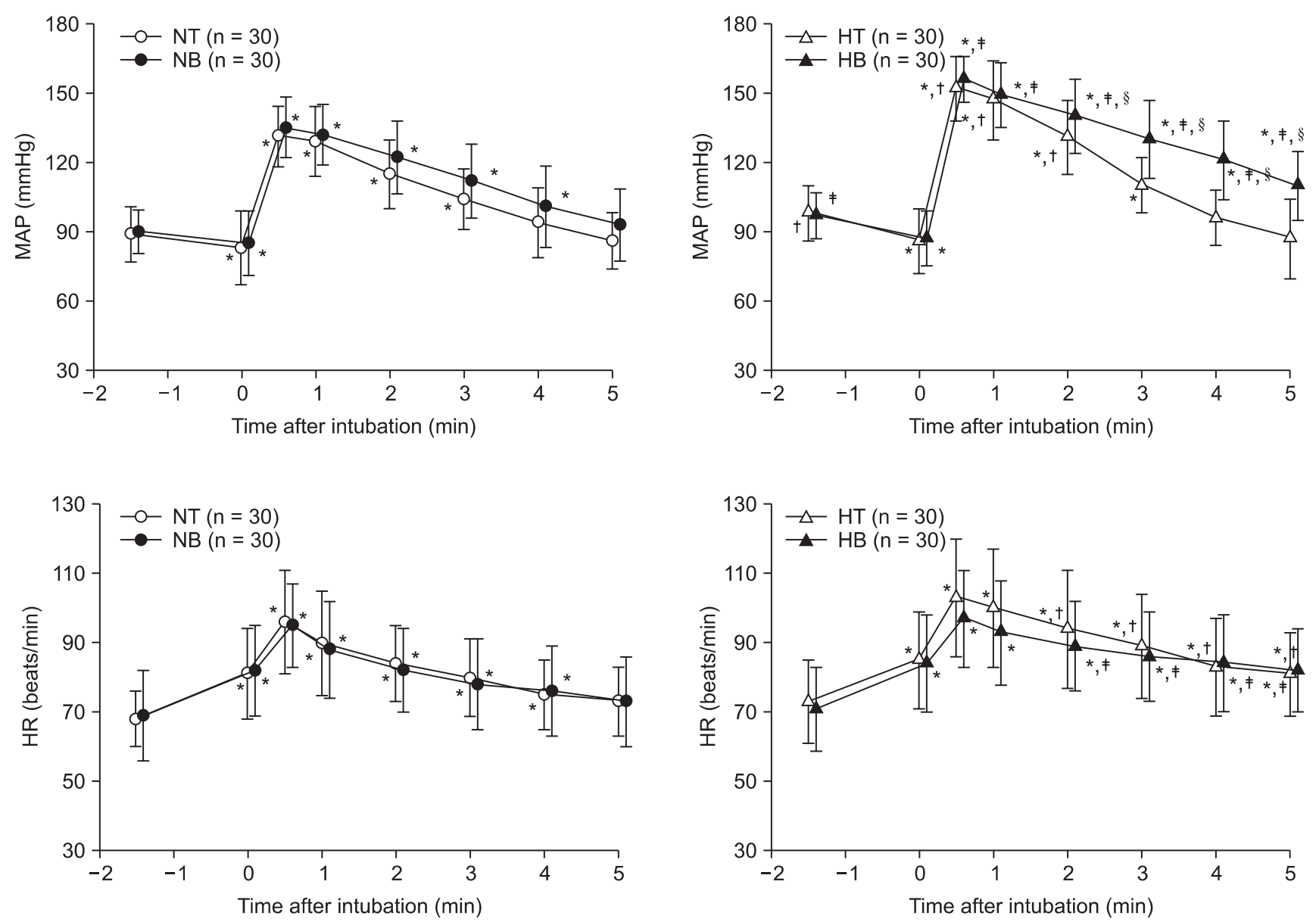

Fig. 1. Mean arterial blood pressure (MAP) and heart rate (HR) in the normotensive (NT and NB groups, left panels) and hypertensive (HT and HB groups, right panels) patients measured before induction of anesthesia $(t=-1.5 \mathrm{~min})$, just before initiating intubation $(\mathrm{t}=0)$, maximum response within $1 \mathrm{~min}$ after intubation, and every $1 \mathrm{~min}$ for first $5 \mathrm{~min}$ after intubation. NT: endotracheally intubated normotensives, NB: endobronchially intubated normotensives, HT: endotracheally intubated hypertensives, HB: endobronchially intubated hypertensives. Values are mean $\pm \mathrm{SD}$. ${ }^{*} \mathrm{P}<0.05$ vs. baseline $(\mathrm{t}=-1.5 \mathrm{~min}),{ }^{\dagger} \mathrm{P}<0.05$ vs. NT, ${ }^{\dagger} \mathrm{P}<0.05$ vs. NB, ${ }^{\S} \mathrm{P}<0.05$ vs. HT. 
endobronchial groups with ( $66 \pm 16 \mathrm{mmHg}$ vs. $68 \pm 12 \mathrm{mmHg}$ ) or without ( $49 \pm 18 \mathrm{mmHg}$ vs. $50 \pm 16 \mathrm{mmHg}$ ) hypertension. However, the maximum increase of MAP from pre-intubation values in the hypertensive groups was significantly greater than that in the normotensive groups in both endotracheal (49 \pm 18 and $66 \pm 16 \mathrm{mmHg}$ in NT and HT groups, respectively) and endobrochial intubation groups (50 \pm 16 and $68 \pm 12 \mathrm{mmHg}$ in $\mathrm{NB}$ and $\mathrm{HB}$ groups, respectively) $(\mathrm{P}<0.05)$ (Fig. 2). The pressor response persisted longer in the HB group than in the HT group $(5.1 \pm 1.6$ vs. $3.2 \pm 0.9 \mathrm{~min}, \mathrm{P}<0.05)$.

HR increased significantly after the induction of anesthesia and further increased by the intubation, the degree of which did not differ among the groups (Fig. 1). However, the tachycardiac response lasted longer in both hypertensive groups than in both normotensive groups ( 4 min vs. longer than $5 \mathrm{~min}, \mathrm{P}<0.05$ ).

The BIS values are illustrated in Fig. 3. Baseline BIS values did not differ among the groups. BIS values significantly decreased after the induction of anesthesia with thiopental in all groups, the degree of which was comparable among the groups. In response to tracheal intubation, BIS values (10-16 BIS units) increased in all groups, being not different among the groups. The maximal increase in BIS was noted within the first 3 min in most cases. The day after the surgery, no patients recalled awareness during anesthesia and intubation.

One patient in the NT, 3 in the NB, 9 in the HT and 11 in the $\mathrm{HB}$ group required an increase of the sevoflurane concentration to treat hypertension or tachycardia. Neither hypotension nor bradycardia was noted, and thus none received either ephedrine or atropine. Premature ventricular contractions appeared immediately after the endobronchial intubation in two patients each in the NB and HB groups. The arrhythmias disappeared spontaneously without treatment.

Plasma catecholamine concentrations before and after intubation are shown in Table 2. Basal levels of norepinephrine and epinephrine were not different among the groups. Induction with thiopental did not affect the plasma concentrations of
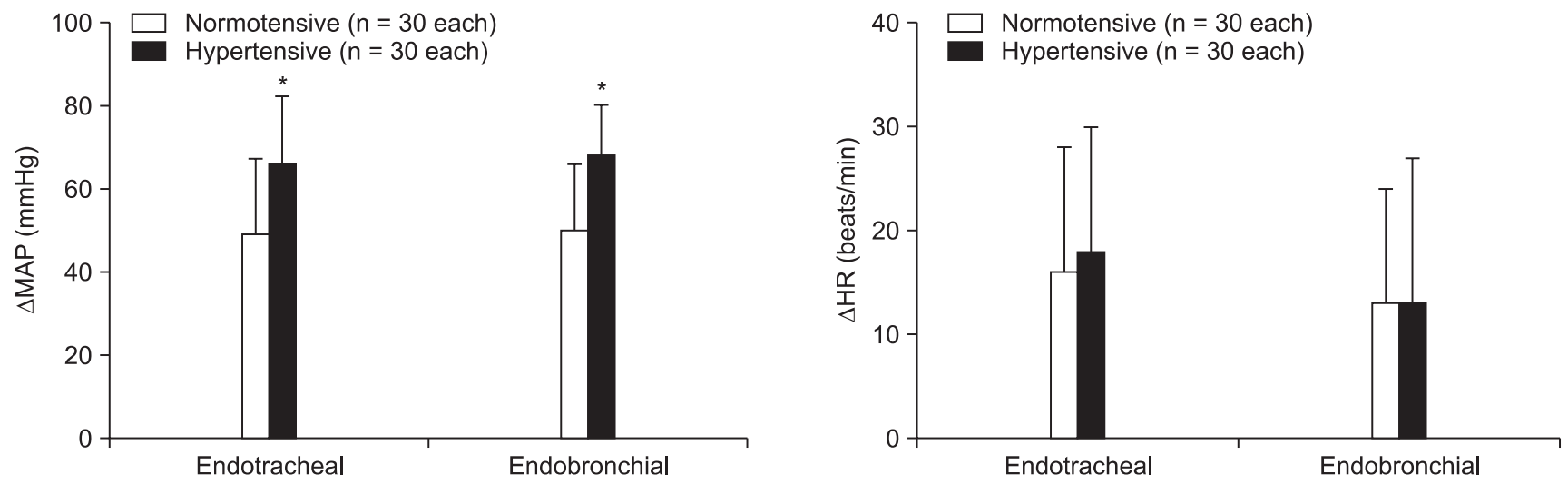

Fig. 2. Changes from pre-intubation to maximum response within $1 \mathrm{~min}$ after intubation in mean arterial pressure $(\Delta \mathrm{MAP})$ and heart rate $(\Delta \mathrm{HR})$ in normotensive and hypertensive patients. Values are mean \pm SD. $* \mathrm{P}<0.05$ vs. normotensive.
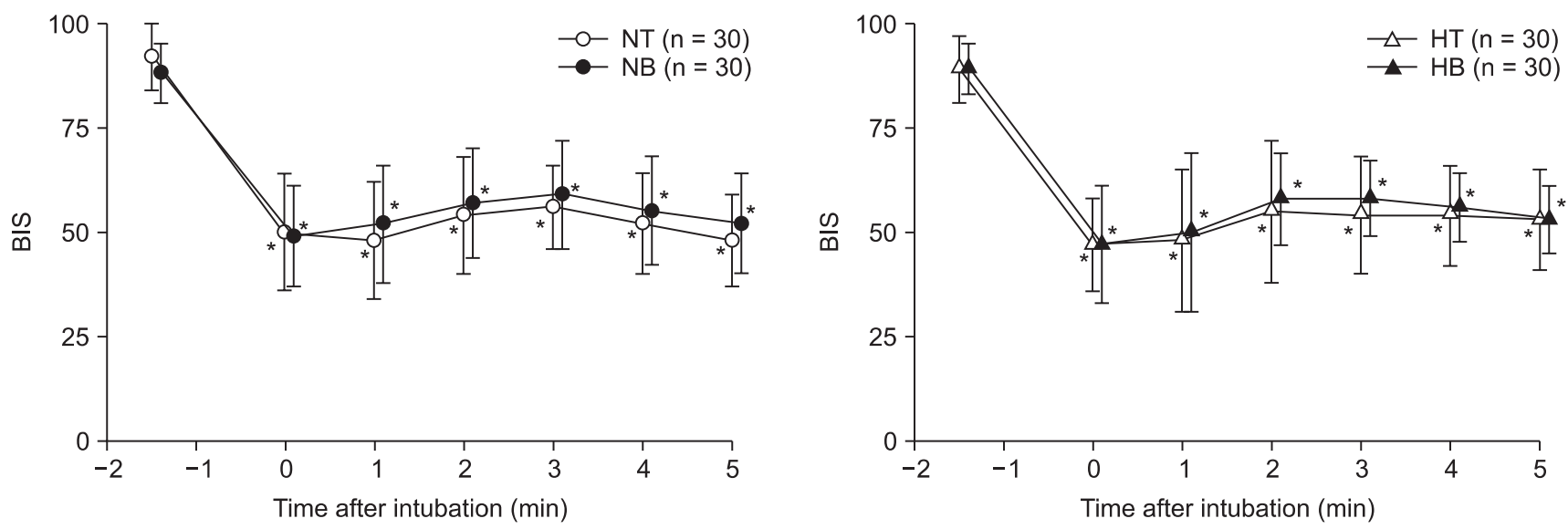

Fig. 3. Bispectral index (BIS) values measured before induction of anesthesia $(t=-1.5 \mathrm{~min})$, just before intubation $(t=0)$, and every 1 min for first 5 min after intubation. Values are mean \pm SD. $* \mathrm{P}<0.05$ vs. baseline $(\mathrm{t}=-1.5 \mathrm{~min})$. 
Table 2. Plasma Catecholamine Concentrations

\begin{tabular}{|c|c|c|c|c|}
\hline & \multicolumn{2}{|c|}{ Normotensive } & \multicolumn{2}{|c|}{ Hypertensive } \\
\hline & NT $(n=30)$ & NB $(n=30)$ & $\mathrm{HT}(\mathrm{n}=30)$ & $\mathrm{HB}(\mathrm{n}=30)$ \\
\hline \multicolumn{5}{|c|}{ Norepinephrine (pg/ml) } \\
\hline Baseline & $216 \pm 102$ & $204 \pm 123$ & $273 \pm 139$ & $227 \pm 132$ \\
\hline Pre-intubation & $215 \pm 86$ & $239 \pm 157$ & $312 \pm 161^{\dagger}$ & $237 \pm 149$ \\
\hline PI-1 & $365 \pm 162^{*, \dagger}$ & $410 \pm 189^{*, \dagger}$ & $652 \pm 305^{* \dagger, \neq, \S}$ & $571 \pm 445^{*,+, \uparrow, \S}$ \\
\hline PI-2 & $326 \pm 146^{*, \dagger}$ & $371 \pm 194^{*, \dagger}$ & $589 \pm 269^{* \dagger, キ, \S}$ & $447 \pm 286^{*, \text {, }}$ \\
\hline PI-5 & $198 \pm 75$ & $263 \pm 148^{*}$ & $333 \pm 139^{\dagger, \uparrow}$ & $313 \pm 160^{\ddagger}$ \\
\hline \multicolumn{5}{|c|}{ Epinephrine (pg/ml) } \\
\hline Baseline & $58 \pm 23$ & $65 \pm 55$ & $61 \pm 45$ & $81 \pm 66$ \\
\hline Pre-intubation & $32 \pm 22^{*}$ & $51 \pm 29$ & $32 \pm 23^{*}$ & $40 \pm 28^{*}$ \\
\hline PI-1 & $59 \pm 57^{\dagger}$ & $83 \pm 59^{\dagger}$ & $46 \pm 37^{\dagger}$ & $110 \pm 121^{*+, \dagger}$ \\
\hline PI-2 & $41 \pm 29$ & $36 \pm 19^{*, \dagger}$ & $28 \pm 18^{*}$ & $46 \pm 40^{*}$ \\
\hline PI-5 & $29 \pm 27^{*}$ & $29 \pm 25^{*, \dagger}$ & $20 \pm 24^{*}$ & $30 \pm 11^{*}$ \\
\hline
\end{tabular}

Values are mean \pm SD or number of patients. NT: endotracheally intubated normotensives, NB: endobronchially intubated normotensives, HT: endotracheally intubated hypertensives, HB: endobronchially intubated hypertensives, Pre-intubation: just before intubation, PI-1, PI- 2 and $\mathrm{PI}-5,1,2$ and $5 \mathrm{~min}$ after the onset of intubation, respectively. ${ }^{*} \mathrm{P}<0.05$ vs. baseline, ${ }^{\dagger} \mathrm{P}<0.05$ vs. pre-intubation values, ${ }^{\dagger} \mathrm{P}<0.05$ vs. NT, ${ }^{\S} \mathrm{P}<$ 0.05 vs. NB.

norepinephrine but significantly decreased epinephrine concentrations. The intubation then caused an increase in norepinephrine concentrations at 1 and $2 \mathrm{~min}$ in all groups, the degree of which was not different between endotracheal and endobronchial intubation in either normotensive or hypertensive patients. However, the degree of increases in norepinephrine concentrations was higher in the hypertensive groups than in the normotensive groups $(\mathrm{P}<0.05)$. Plasma epinephrine concentrations were also increased significantly after intubation in each group, however, the degree of which was not different among the four groups.

\section{Discussion}

Our study demonstrated that the peak hemodynamic and BIS changes were not affected by the method of intubation (endotracheal vs. endobronchial intubation) regardless of the presence or absence of hypertension, although the pressor response lasted longer in the HB than in the HT group (5.1 min vs. $3.2 \mathrm{~min}$ ). However, the hypertensive patients (HT and HB groups) exhibited more pronounced pressor and catecholamine responses than the normotensive (NT and NB groups). This finding is consistent with that in a previous study [1], which demonstrated pronounced pressor responses in the hypertensives compared with the normotensives.

It has been reported that the distribution of airway receptors is richest in the larynx, being especially concentrated in the proximal part of the tracheobronchial tree $[14,15]$. Moreover, these receptors become more chemosensitive and less mechanosensitive in the distal portion [16]. Consequently, no difference in pressor and catecholamine responses has been demonstrated between laryngoscopy with and without endotracheal intubation in healthy patients [2]. Therefore, the stress response to endobronchial intubation associated with a long way insertion of DLT up to bronchus, and carinal and lower airway stimulation on placement is unlikely to affect the hemodynamic outcomes.

The magnitude of stimuli to the upper airway during anesthesia induction and intubation depends on the duration of intubation [17] and the force exerted on the tongue during laryngoscopy [18]. Direct laryngoscopy that takes less than 15 seconds is known to minimize pressor responses [17]. In the present study, it took significantly longer by the endobronchial intubation than by the endotracheal intubation $(\sim 23 \mathrm{~s}$ vs. $\sim 14 \mathrm{~s}, \mathrm{P}<0.01)$. Although we did not measure forces applied on tongue during laryngoscopy, DLT may have caused more force because of its much larger size compared to SLT. Therefore, a small difference in the intubation time $(<10 \mathrm{~s})$ and the force exerted on the tongue between endotracheal and endobronchial intubation may have a little impact on peak hemodynamic and catecholamine responses to intubation. The longer duration of pressor responses rather than their peak values in patients with hypertension may be related to a longer time frame necessary to accomplish the endobronchial intubation and more force applied on the tongue.

It has been well known that the pressor response to intubation is proportional to plasma norepinephrine levels [19]. Indeed, the magnitude of increases in norepinephrine concentrations was significantly greater along with the pressor response in the hypertensive than in the normotensive in the present study. However, neither the norepinephrine levels nor the hemodynamic parameters were significantly different between the SLT and DLT groups, suggesting that the magnitude of the stress response between the groups is comparable. The 
epinephrine concentrations increased significantly in all groups, of which magnitude was not affected by the hypertension. The epinephrine response is unlikely to reflect the exaggerated pressor response in patients with hypertension.

Patients who require DLT endobronchial intubation are mostly elderly, in whom cardiovascular instability may contribute to perioperative myocardial ischemia and cardiac morbidity $[5,6,20]$. In the present study, the pressor responses were more pronounced and tachycardiac responses prolonged in the hypertensive compared with those in the normotensive regardless of the method of intubation. We observed that hypertensive patients (HT and HB) received additional sevoflurane more frequently to treat hypertension or tachycardia compared with the normotensives. In addition, the HB group showed a prolonged pressor response compared with the HT group. The endobronchial intubation is often achieved not easily in one attempt. Moreover, the fiberoptic bronchoscopy to confirm proper placement of DLT may stimulate airway, resulting in a prolonged pressor response. Thus, the maintenance of hemodynamic stability during endobronchial intubation is of particular clinical importance in elderly patients with hypertension.

BIS $\leq 60$ is widely used as an end-point of hypnosis during general anesthesia [21]. BIS values after the intubation remained below 60 in all groups, indicating a low probability of recall and conscious perception [22]. Since EEG responses to noxious stimulation may occur when the depth of anesthesia is changed [23] and the depth of anesthesia in turn depends on the intensity of the noxious stimulation [24], we postulated that the endobronchial intubation should cause a greater BIS change and hence the risk of awareness than the endotracheal intubation. However, BIS values at the moment of intubation did not differ between the endotracheal and endobronchial groups. In addition, no one recalled awareness during anesthesia and intubation in either group. The same amount of thiopental was used to induce anesthesia in the two intubation groups, not differently affecting the risk of awareness.

This study has a few limitations. First, although opioids are commonly given with thiopental during the induction of anesthesia, we did not use them in order not to influence the results of our study. Therefore, the depth of anesthesia was probably inadequate during laryngoscopy and intubation. However, for hypertension (an increase of SAP $>30 \%$ above baseline for $>60 \mathrm{~s}$ ) or tachycardia (HR $>120$ beats $/ \mathrm{min}$ for $>60 \mathrm{~s}$ ) associated with intubation, the inspired sevoflurane concentration was increased in increments of $1 \%$ in the present study. Second, the use of sevoflurane as a rescue medication during induction may have influenced the hemodynamic responses in the present study. However, since the number of patients who received sevoflurane between endotracheal and endobronchial groups with (9 in HT vs. 11 in HB) or without hypertension ( 1 in NT vs. 3 in NB) was similar, and sevoflurane was given after but not before intubation, the use of sevoflurane is unlikely to have influenced our results. Third, our study population had an average age of approximately 68 years and did not have difficult airways. Our results may not apply to different age groups and to patients with difficult airways. Finally, collection of hemodynamic and BIS data was not performed in a blinded fashion. Ideally, the investigator who recorded the data would not have known the type of intubation tube. However, since many of the observations are objective (e.g., BIS value, MAP, HR), and the investigator was not involved in the intraoperative care, a lack of intraoperative blinding is unlikely to have influenced our results.

In summary, the endobronchial intubation caused similar degrees of hemodynamic and arousal responses, as measured by BIS, as the endotracheal intubation regardless of the presence or absence of hypertension. However, the magnitude of pressor and catecholamine responses was greater in the hypertensive than in the normotensive patients.

\section{References}

1. Prys-Roberts C, Greene LT, Meloche R, Foëx P. Studies of anaesthesia in relation to hypertension. II. Haemodynamic consequences of induction and endotracheal intubation. Br J Anaesth 1971; 43: 53147.

2. Shribman AJ, Smith G, Achola KJ. Cardiovascular and catecholamine responses to laryngoscopy with and without tracheal intubation. Br J Anaesth 1987; 59: 295-9.

3. Wilder-Smith OH, Hagon O, Tassonyi E. EEG arousal during laryngoscopy and intubation: comparison of thiopentone or propofol supplemented with nitrous oxide. Br J Anaesth 1995; 75: 441-6.

4. Hamaya Y, Dohi S. Differences in cardiovascular response to airway stimulation at different sites and blockade of the responses by lidocaine. Anesthesiology 2000; 93: 95-103.

5. Mangano DT. Perioperative cardiac morbidity. Anesthesiology 1990; 72: 153-84.

6. Harris T, Cook EF, Kannel W, Schatzkin A, Goldman L. Blood pressure experience and risk of cardiovascular disease in the elderly. Hypertension 1985; 7: 118-24.

7. Scott JC, Stanski DR. Decreased fentanyl and alfentanil dose requirements with age. A simultaneous pharmacokinetic and pharmacodynamic evaluation. J Pharmacol Exp Ther 1987; 240: $159-66$.

8. Minto CF, Schnider TW, Egan TD, Youngs E, Lemmens HJ, Gambus $\mathrm{PL}$, et al. Influence of age and gender on the pharmacokinetics and pharmacodynamics of remifentanil. I. Model development. Anesthesiology 1997; 86: 10-23.

9. Latson TW, Ashmore TH, Reinhart DJ, Klein KW, Giesecke AH. Autonomic reflex dysfunction in patients presenting for elective surgery is associated with hypotension after anesthesia induction. 
Anesthesiology 1994; 80: 326-37.

10. Thompson JP, West KJ, Hill AJ. The cardiovascular responses to double lumen endobronchial intubation and the effect of esmolol. Anaesthesia 1997; 52: 790-4.

11. Hannallah MS, Benumof JL, Ruttimann UE. The relationship between left mainstem bronchial diameter and patient size. J Cardiothorac Vasc Anesth 1995; 9: 119-21.

12. Holly JM, Makin HL. The estimation of catecholamines in human plasma. Anal Biochem 1983; 128: 257-74.

13. Brice DD, Hetherington RR, Utting JE. A simple study of awareness and dreaming during anaesthesia. Br J Anaesth 1970; 42: 535-42.

14. Widdicombe JG. Pulmonary and respiratory tract receptors. J Exp Biol 1982; 100: 41-57.

15. Sant'Ambrogio G. Nervous receptors of the tracheobronchial tree. Annu Rev Physiol 1987; 49: 611-27.

16. Karlsson JA, Hansson L, Wollmer P, Dahlbäck M. Regional sensitivity of the respiratory tract to stimuli causing cough and reflex bronchoconstriction. Respir Med 1991; 85 Suppl A: 47-50.

17. Stoelting RK. Circulatory changes during direct laryngoscopy and tracheal intubation: influence of duration of laryngoscopy with or without prior lidocaine. Anesthesiology 1977; 47: 381-4.

18. Bucx MJ, van Geel RT, Scheck PA, Stijnen T. Cardiovascular effects of forces applied during laryngoscopy. The importance of tracheal intubation. Anaesthesia 1992; 47: 1029-33.

19. Derbyshire DR, Chmielewski A, Fell D, Vater M, Achola K, Smith G. Plasma catecholamine responses to tracheal intubation. Br J Anaesth 1983; 55: 855-60.

20. Fox EJ, Sklar GS, Hill CH, Villanueva R, King BD. Complications related to the pressor response to endotracheal intubation. Anesthesiology 1977; 47: 524-5.

21. Katoh T, Suzuki A, Ikeda K. Electroencephalographic derivatives as a tool for predicting the depth of sedation and anesthesia induced by sevoflurane. Anesthesiology 1998; 88: 642-50.

22. Myles PS, Leslie K, McNeil J, Forbes A, Chan MT. Bispectral index monitoring to prevent awareness during anaesthesia: the B-Aware randomised controlled trial. Lancet 2004; 363: 1757-63.

23. Kaada BR, Thomas F, Alnaes E, Wester K. EEG synchronization induced by high frequency midbrain reticular stimulation in anesthetized cats. Electroencephalogr Clin Neurophysiol 1967; 22: 220-30.

24. Kissin I, Stanski DR, Brown PT, Bradley EL Jr. Pentobarbitalmorphine anesthetic interactions in terms of intensity of noxious stimulation required for arousal. Anesthesiology 1993; 78: 744-9. 